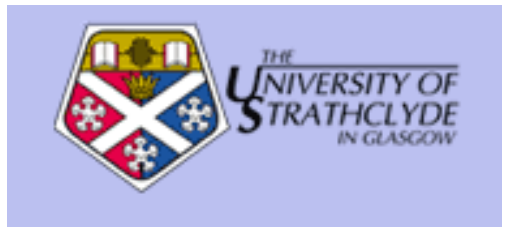

Porta, S. and Crucitti, P. and Latora, V. (2008) Multiple centrality assessment in Parma: a network analysis of paths and open spaces. Urban Design International, 13 (1). pp. 41-50. ISSN 1357-5317

http://strathprints.strath.ac.uk/18468/

This is an author produced version of a paper published in Urban Design International, 13 (1). pp. 41-50. ISSN 1357-5317. This version has been peer-reviewed but does not include the final publisher proof corrections, published layout or pagination.

Strathprints is designed to allow users to access the research output of the University of Strathclyde. Copyright (C) and Moral Rights for the papers on this site are retained by the individual authors and/or other copyright owners. You may not engage in further distribution of the material for any profitmaking activities or any commercial gain. You may freely distribute both the url (http://strathprints.strath.ac.uk) and the content of this paper for research or study, educational, or not-for-profit purposes without prior permission or charge. You may freely distribute the url (http://strathprints.strath.ac.uk) of the Strathprints website.

Any correspondence concerning this service should be sent to The Strathprints Administrator: eprints@cis.strath.ac.uk 


\title{
MULTIPLE CENTRALITY ASSESSMENT IN PARMA A NETWORK ANALYSIS OF PATHS AND OPEN SPACES
}

\author{
SERGio PORTA ${ }^{\mathrm{a}}$, PAOLO CRUCITTI $^{\mathrm{b}}$, Vito LATORA $^{\mathrm{c}}$ \\ a Dipartimento di Progettazione dell'Architettura, Politecnico di Milano, Italy, sergio.porta@polimi.it \\ ${ }^{\mathrm{b}}$ Scuola Superiore di Catania, Italy, pacrucitti@ssc.unict.it \\ ${ }^{\mathrm{c}}$ Dipartimento di Fisica e Astronomia, Università di Catania and INFN Sezione di Catania, Italy, vito.latora@ct.infn.it
}

\begin{abstract}
One of the largest of Europe, the recently realized University Campus "Area of the Sciences" in Parma, northern Italy, has been planned for a comprehensive programme of renovation and revitalization with a special focus on vehicular accessibility and the quality of open spaces. As part of the problem setting, Multiple Centrality Assessment (MCA) - a process of network analysis based on primal graphs, a set of different centrality indexes and the metric computation of distances - has been implemented in order to understand why the existent networks of open spaces and pedestrian paths are scarcely experienced by students as well as faculty and staff members and appear so poorly integrated with the life on Campus. MCA has also given a relevant contribution to the comparative evaluation of two proposed scenarios, leading to the identification of one final solution of urban design.

In the present paper the first professional application of MCA, an innovative approach to the network analysis of geographic complex systems, is presented and its relevance in the context of a problem of urban design illustrated.
\end{abstract}

\section{Introduction}

The science of networks has been witnessing a rapid development in recent years since the seminal work of Watts and Strogatz on the so-called "small worlds" in 1998 [1]: the metaphor of the network has been applied to complex, self-organized systems as diverse as social, biological, technological and economic, leading to several unexpected results [2]. In particular, the issue of centrality in networks has remained pivotal, since its introduction in a part of the studies of humanities named structural sociology [3]. The idea of centrality was first applied to human communication by Bavelas $[4,5]$ who was interested in the characterization of the communication in small groups of people and assumed a relation between structural centrality and influence/power in group processes. Since then various measures of structural centrality have been proposed over the years to quantify the importance of an individual in a social network. Currently, centrality is a fundamental concept in network analysis though with a different purpose: while in the past the role and identity of central nodes was investigated, now the emphasis is more shifted to the distribution of centrality values through all nodes: centrality, as such, is treated like a shared resource of the network "community" - like wealth in nations - rather than the unique property of the excellent.

In urban planning and design centrality - though under different terms like "accessibility", "proximity", "integration", "connectivity", "cost", "effort" - has entered the scene stressing the idea that some places (or streets) are more important than others because they are more central [6]. A pioneering discussion of centrality in the analysis of spatial systems has been successfully operated after Hillier and Hanson seminal work on cities since the mid Eighties [7]. Space Syntax, the related methodology of urban analysis, has been raising growing evidence of the correla- tion between the level of "integration" of urban spaces - a closeness centrality in all respects - and phenomena as diverse as crime rates, pedestrian and vehicular flows, retail commerce vitality and human wayfinding capacity [8]. However, the Space Syntax approach is profoundly different from most previous uses of networks in geographic space, i.e. in transportation and land use planning or economic geography: in fact, while in those studies the urban pattern is subjected to a primal translation in a graph, where streets are turned into edges and intersections into nodes, Space Syntax follows the dual representation where streets are turned into nodes and intersections into edges. This dual character leads Space Syntax to the abandonment of metric distance (one street is one point no matter its real length) - which, conversely, was the core of those territorial studies - and to actually reach the topologic world of non geographic systems. Moreover, the Space Syntax analysis is mainly based on just one centrality index, called "integration", which can be used only in association with a generalization model in order to minimize the so called "edge-effect", a typical distortion of the spatial distribution of centrality values that groups higher scores around the centre of the image no matter the actual configuration of the network $[9,10]$.

In order to overcome such pitfalls, we have recently proposed, in three related publications $[11,12,13]$ an innovative procedure for the newtork analysis of geographic systems called Multiple Centrality Assessment (MCA). An extensive discussion of primal graph construction and MCA in the context of both the new science of networks and Space Syntax has been provided in the latter publication and is hereby summarized in the next chapter; the present paper, in fact, mainly focuses on a first application of MCA to a real problem of urban design in Parma, northern Italy, which is the subject of chapter 3. 


\section{Primal graph construction and Multiple Centrality Assessment (MCA): a summary}

MCA is a structured process aimed at the evaluation of the spatial distribution of centrality over geographic systems like systems of urban streets or spaces.

The first step in operating MCA is the translation of the spatial system into a graph which is a mathematical entity defined by a set $N$ of nodes and a set $K$ of links, or edges connecting pairs of nodes. Roughly speaking, a street (or spatial) pattern is translated in a primal graph so that intersections (or places) are turned into nodes and streets (or connections) into edges. Networks of streets and intersections are graphed under a "road-centerline-betweennodes" rule: one edge is defined by just two end-nodes (from-node and to-node) and a variable number of vertexes (points of linear discontinuity); edges follow the geographical footprint of real streets as they appear on the original map; links between two edges are always located at one of the two defining nodes (intersections); the distance between two nodes is calculated metrically along the edge, following real curves and angles. Thus, streets are identified directly on the original map, with no use of any generalization model.

Networks of places and connections are analogous to those of street and intersections. In the present work for Parma, places and connections have been given a conventional definition: a place is an open space characterized by: 1. a convex internal shape; and 2. visible borders (building facades, tree lines, hedges, pavement changes, paths, fencings...) on at least three quarters of the perimeter; and 3. a minor dimension $<70$ metres. Two places are connected when: 1 . the minor distanced between their borders is $<35$ metres; and 2 . they are not separated by physical barriers (main trafficked streets, closed fencings, buildings, floods, walls, impassable level gaps...).

On such kind of graphs, MCA investigates how centrality "flows" through edges over nodes. A key feature of MCA is that centrality is considered a multifold concept; in short, we have many centralities depending on what is our notion of "being central". Heavily drawing from the mentioned studies in structural sociology, we acknowledge four different families of "being central", each described by a different centrality index: 1 . "being central as being close to others", closeness centrality $\left(C^{C}\right) ; 2$. being central as being between others", betweenness centrality $\left(B^{C}\right)$; 3. "being central as being straight to others", straightness centrality $\left(S^{C}\right)$; 4. "being central as being critical for all others as a group", information centrality $\left(I^{C}\right)$. Again, we forward the readers to our previous work for a formal definition of those indexes.

Finally, in MCA all distances are measured metrically; thus, the distance between nodes $i$ and $\mathrm{k}$ is, say, 425.32 metres. This metric/geographic concept of distance is used in MCA for all computations, including the identification of shortest paths, or "geodesics", between pairs of nodes.

All that deeply differentiates MCA from Space Syntax, which conversely is based on dual graphs, the preliminary use of a generalization model (axial mapping), and just one main centrality index (the "integration" centrality, which is a closeness centrality in all respects). Moreover, like all the dual analysis, Space Syntax is necessarily an- chored to a topologic concept of distance where the distance between nodes $i$ and $k$ is, say, 3 steps. With all the different meanings that have been assigned to the word "step" in recent literature, as street intersection [14], binary direction change [15], gradual direction change [16], characteristic point [17], street-name change [18], stepdistance has to be recognized as a direct, unavoidable outcome of the dual approach.

General advantages of MCA, if compared to dual approaches like Space Syntax, are multifold: 1. it is not based on any generalization model, therefore is more legible, feasible and objective; 2 . it is fit to access the huge amount of information resources developed under the road-centreline-between-nodes world standard, including network constructed for traffic engineering and modelling or geo-mapping worldwide; 3 . it is more realistic, in that it is grounded on metric rather than on step-distance measurements; 4. it gives a set of multifaceted pictures of reality, one for each centrality index, rather than just one: that leads to more argumentative, thus less assertive, indications for action. Moreover, from a pure research perspective, MCA makes it possible to analyse geographic networks under the same roof of well established studies of non-geographic ones such as biologic, technological or social networks.

Let's see how MCA helped in dealing with the open spaces decay in the University Campus "Area of the Sciences" in Parma.

\section{MCA: the spatial analysis of the University Campus "Area of the Sciences" in Parma}

\subsection{A case's outline}

The Campus "Area of the Sciences" of the University of Parma has been developed in the last two decades on 77 ha of land at the outskirt of the city of Parma, over a plain area at the bottom of the first Appennini hills. The site development follows a general plan conceived with the aim of ensuring the largest possible autonomy to each department, resulting in an addition of self-containing sites, each served by its own parking and accessibility facilities in a huge but poorly maintained and mainly uncontrolled "green" space. At the geometrical center of the site a huge technologic plant is located, while services for food and rest were decentralized to the southern and western fringe of the area. Sport facilities for football, tennis, rugby, golf and gym have been realized over the years in the north western side of the Campus.

Currently, the Campus is used by some 8.600 students and other 980 staff or faculty members on a daily basis. After a period of intense development, a political impulse has been given for solving two growing problems that are seen as main threats to the further attractiveness and functionality of Campus: 1 . the problem of accessibility: vehicular traffic and parking has never been really ruled, resulting in aggressive behaviors against open spaces and non-motorized uses everywhere on Campus; 2. Public paths and open spaces that cover a large part of the area are nevertheless poorly used and maintained, often prone to abandonment or improper activities. 
A project was requested to face such problems and suggest possible solutions. The project envisions integrated actions for both a new model of access and the revitalization of open spaces. In the following, we offer a short summary of the former and a more extensive illustration of the latter, that was based on MCA.

\subsection{The problem of accessibility: a short summary}

The rationale for the betterment of accessibility conditions on Campus is the shift from an "everyoneeverywhere" model to a selected users in "selected places" one. That can be pursuit at the condition of a deep change in parking habits: a limited increase in parking offer is obtained after a large increase of short-time parking and a almost analogous decrease of long-time parking, where the former has been mostly located in central areas, adjacent to the departments, and the latter in outer areas.

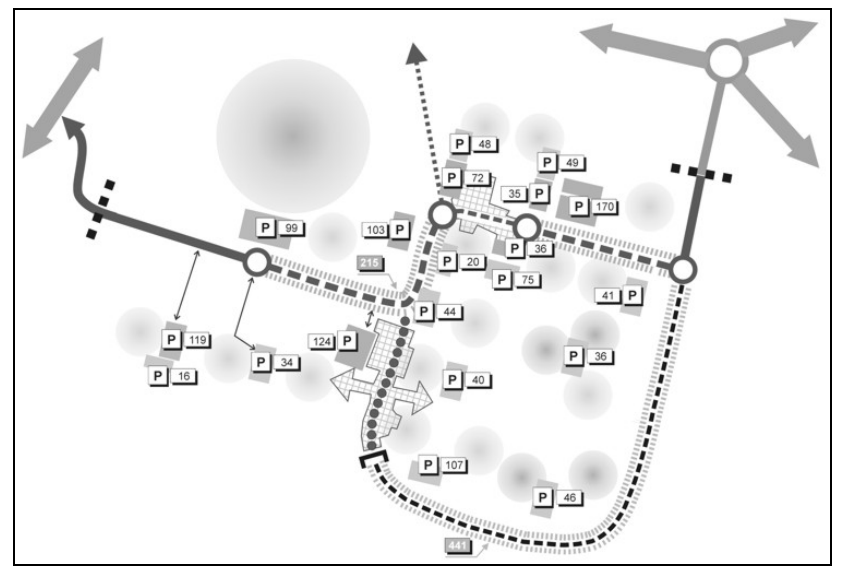

Fig. 1

The conceptual plan of the envisioned development of accessibility facilities on Campus: an organic whole substitutes a collection of self-contained, inward-looking settlements while large parts of central areas are freed from in-lot parking, so made available for connecting landscaped environments.

On-street parking largely substitutes parking areas, especially along main streets: on-street parking, in fact, contributes to a broader policy of traffic calming on main streets, which have been found dangerous due to the over limits speeds of significant shares of passing vehicles. In so doing, large open areas in central locations that currently work as barriers are turned in new landscaped areas available for high quality connecting facilities like cycle/pedestrian paths, rest areas or visual corridors.

In two parts of the main street pattern that have been found critical for the continuity of cycle/pedestrian paths across the whole Campus, the streetscape has been radically converted to pedestrian oriented uses and vehicular uses have been particularly constrained.

Policies for establishing the bicycle as the prime means for internal trips on Campus have been suggested: University owned bicycle facilities have been designed along the outer routes of access and long-time parking, anchored to a system of bicycle parking and free-rent that covers the whole area.
The overall effect of accessibility measures is that a collection of independent parts are turned into one sole organism and alternative means of transportation, especially for internal trips, are fostered.

\subsection{The problem of open space's livability}

The problem of the decay of open spaces on Campus has been investigated posing a special attention to the topological relationships that link each constituent part to every other in the system.

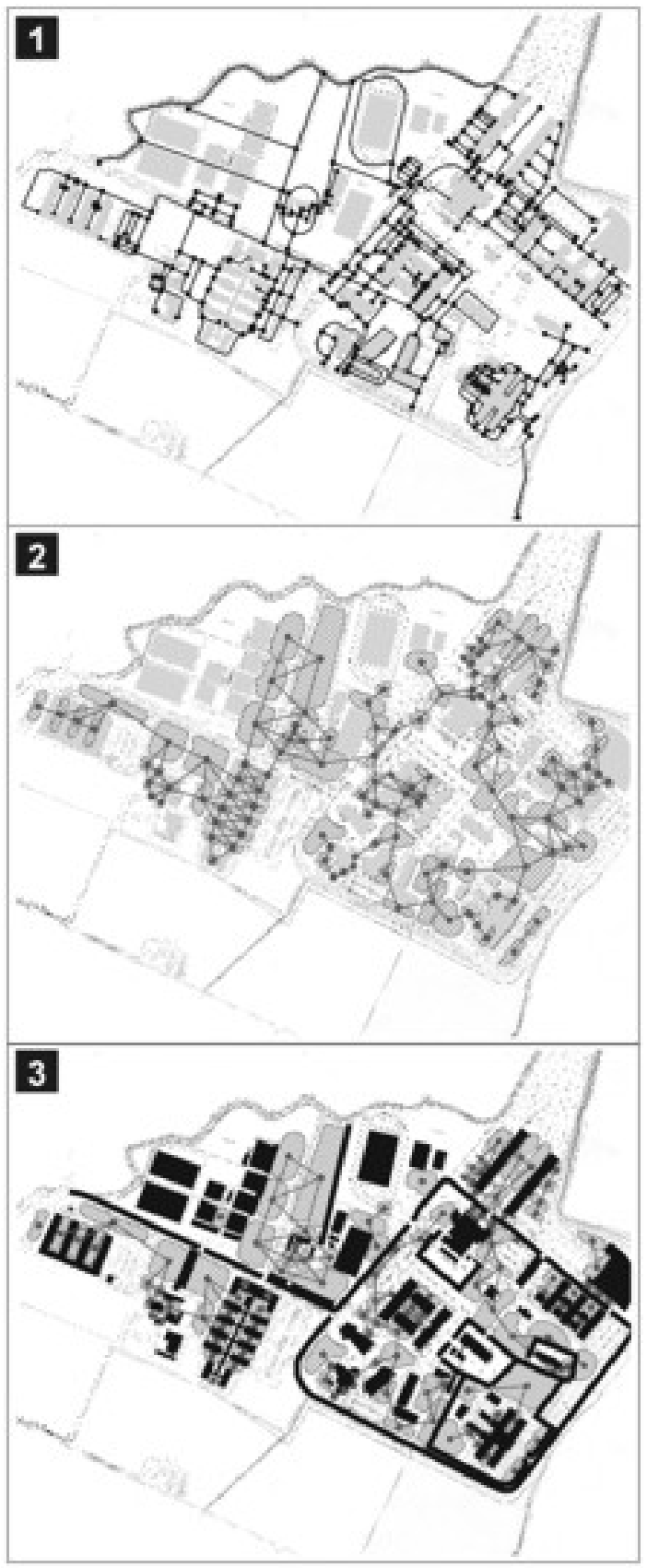

Fig. 2

The three primal graphs constructed in the first step of MCA analysis: 1 . Paths: the network of cycle/pedestrian paths and intersections; 2. Potential places: the network of places and connections in absence of barriers (main trafficked streets, buildings, fencings...); 3. Real places: the network of places and connections after barriers (black) have been considered. 
Consequently, the MCA analysis has firstly constructed three different graphs (fig. 2): 1. the newtork of cycle/pedestrian paths; 2. the "potential" newtork of places; 3 . the "real" network of places. The difference between the potential and the real networks of places is that in the former all barriers have been artificially cancelled, as if all places were "freely" disposed on a plain and uninterrupted space. The introduction of the potential network is aimed at evaluating, by comparison, the impact of existent barriers like buildings or fencings on the connectivity of spaces and their structure of centrality.

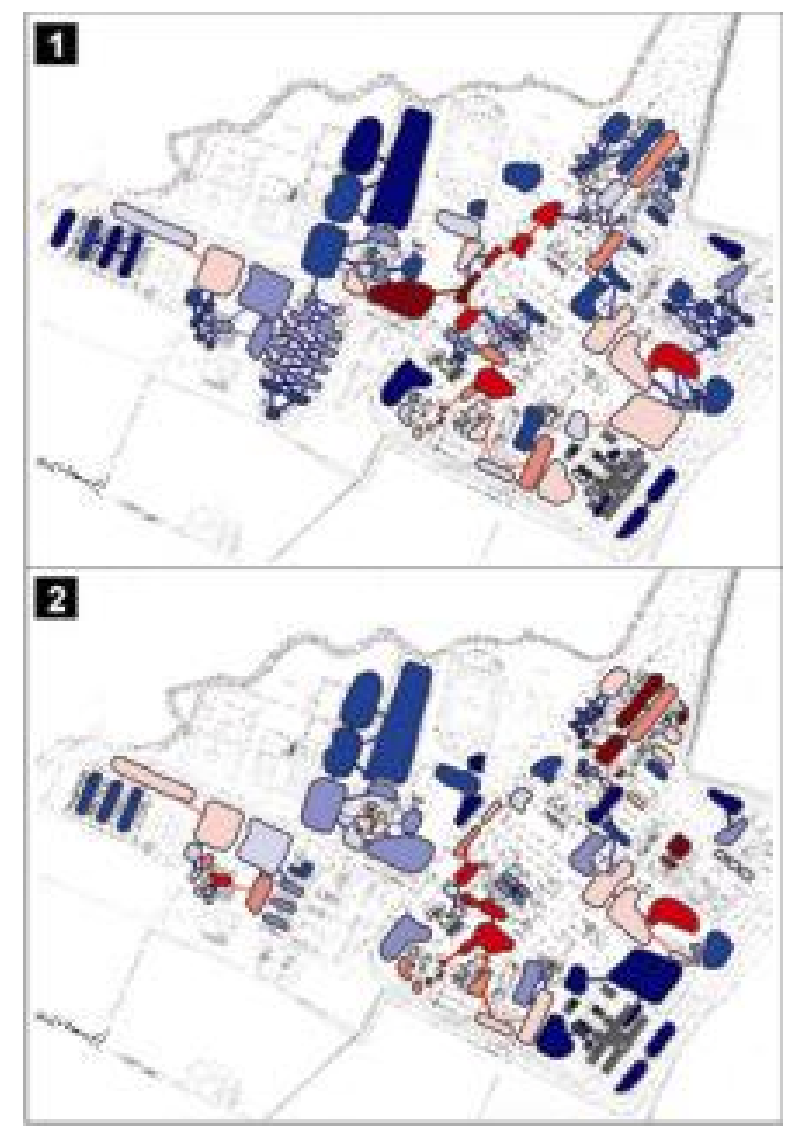

Fig. 3

$\mathrm{I}^{\mathrm{C}}$ distribution over the network of places and connections; 1 . the "potential" network, where physical barriers are not considered; 2. the "real" network, where barriers are considered.

The real network (2) is fragmented in four disconnected subnetworks, each one with its own focal spot; all these "local centres" do not form any organic system.

The potential network (1) is quite different, a single connected network with one emergent centrality located near the cultural centre and the sport facilities.

In all cases, the structure of places and that of paths (see fig. 5, col. 1) are not consistent one the other, in that marginal as a general rule paths serve central places and central paths serve marginal places.

The real network of places (fig. $3_{1}$ ) is a fragmented system made of four disconnected sub-networks; each subnetwork exhibits an autonomous structure of centrality around four independent "local centers". All these local centers are located well detached from the distributor road, while close to the buildings of scientific departments or services.

This structure does not find any correspondence with the structure of centralities on the network of existent paths (fig. 5, col. 1): in this case, paths located along the distributor road are the most central, while internal paths located close to buildings and facilities exhibit a significant drop in centrality values. The only exception to this pattern emerges with closeness centrality: here, we see that internal paths that are central in the sense that they are more close to all others in the network $\left(\mathrm{C}^{\mathrm{C}}\right)$ are not so central in other senses, especially as being the intermediaries among other relationships $\left(\mathrm{C}^{\mathrm{B}}\right)$ or as being critical for the connectivity of the whole system $\left(\mathrm{I}^{\mathrm{C}}\right)$.

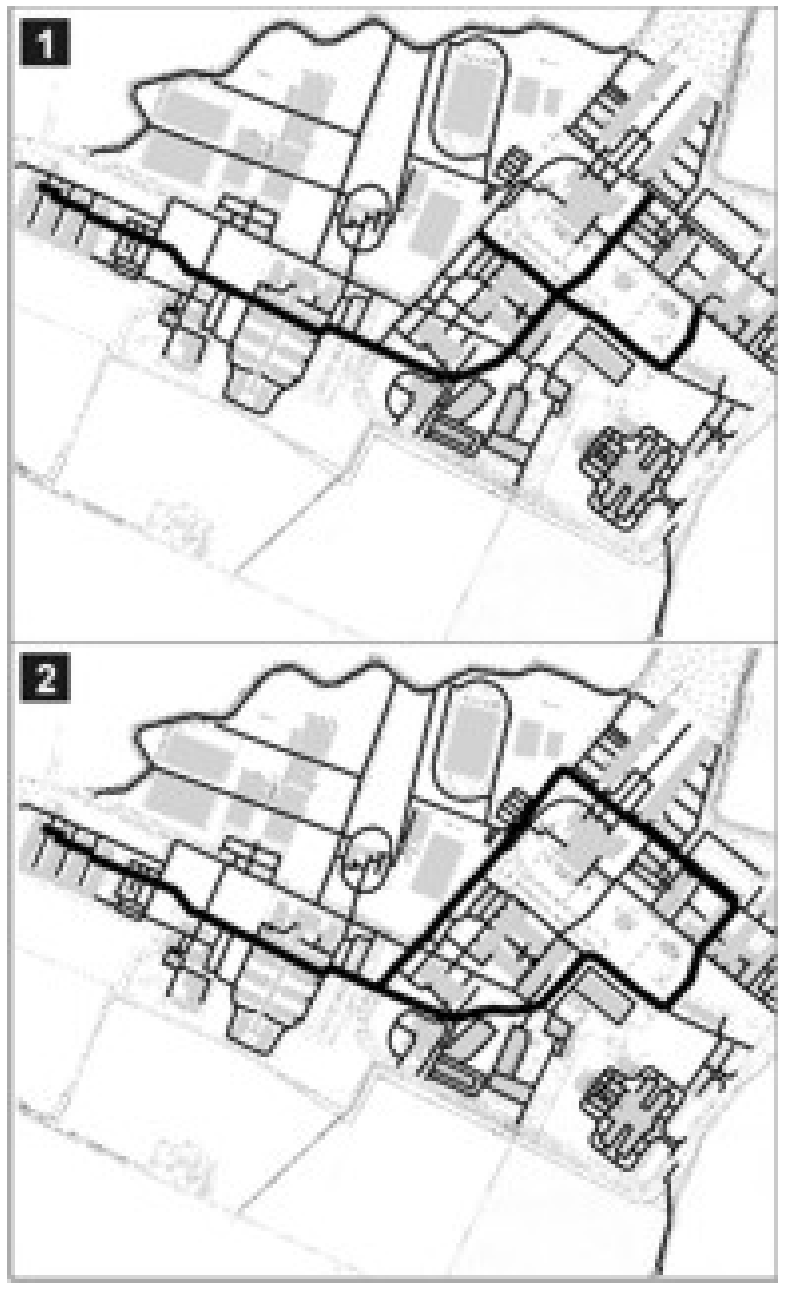

Fig. 4

The proposed new central routes (thick black) for the system of cycle/pedestrian paths: 1. Scenario A: the central spine; 2. Scenario $\mathrm{B}$ : the ring. After both scenarios were compared through a dedicated MCA analysis (see fig. 4, col. 2 and 3), the former spine-shaped route was selected for the new urban design plan.

The inconsistency between the structures of centrality over the two systems of places and paths seems a major obstacle to the functioning of the open spaces on Campus in terms of social use. 


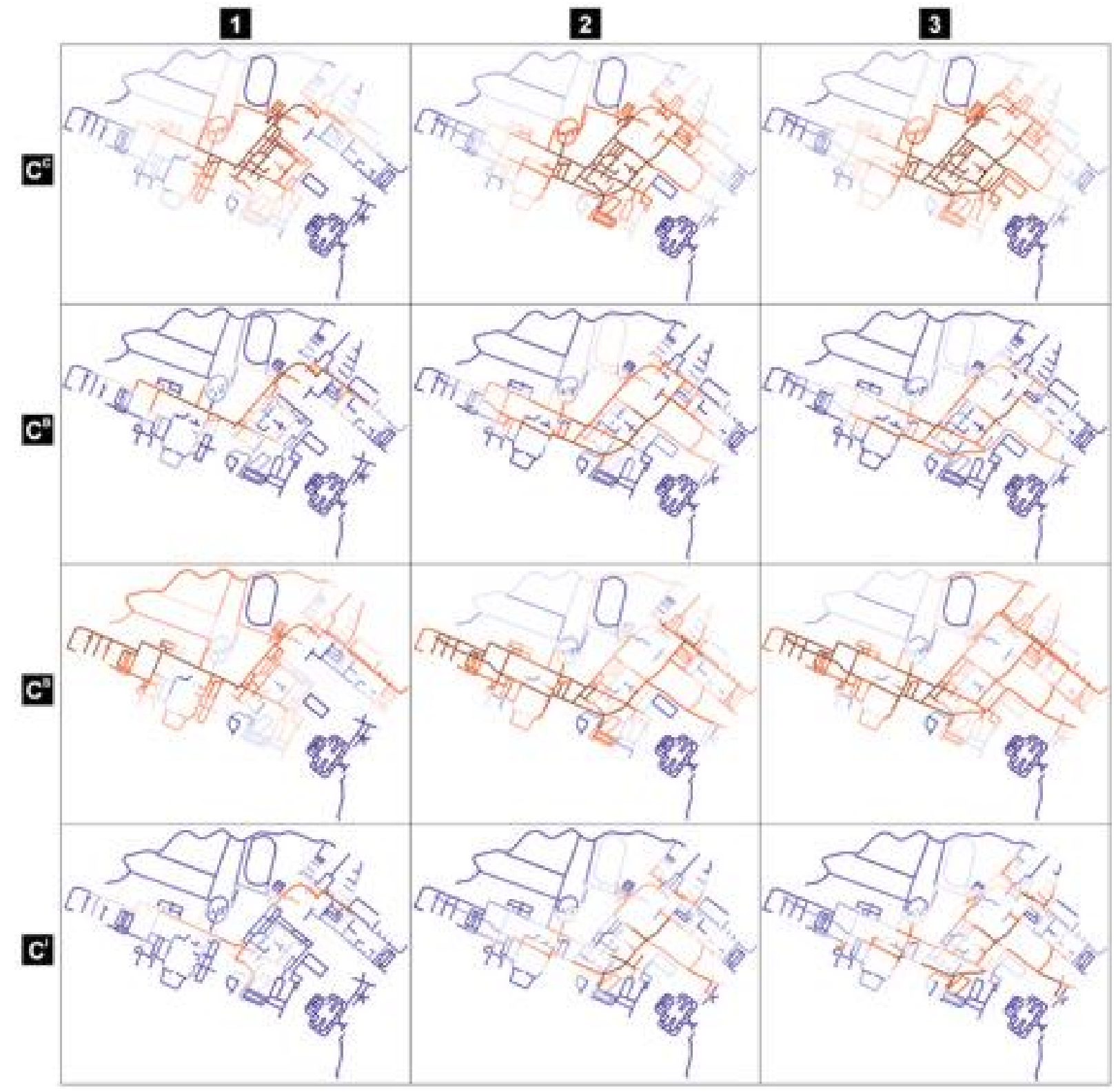

Fig. 5

Centrality distributions over the network of cycle/pedestrian paths and intersections; 1. the existent network; 2. the proposed network, scenario A: the central spine; 3. the proposed network, scenario B: the ring.

The $\mathrm{C}^{\mathrm{C}}$ distribution appears in all cases deeply affected by the "edge effect", the distortion that typically groups higher $\mathrm{C}^{\mathrm{C}}$ scores around the centre of the image rather than revealing any structural order of the network. In this case, however, the borders of the image do express a relevant territorial meaning, because the Campus is really self-centred and isolated at the fringe of the urban settlement, a peripheral "island" with just two points of connections with the larger system of the city; so in this specific case the picture emerging from the analysis of $\mathrm{C}^{\mathrm{C}}$ centrality index represents much more an inherent feature of the real network of the Campus than an artificial outcome of the borders' cut.

In the existent network (col. 1), a spine of higher centrality emerges along the distribution road, which is a mostly vehicular channel that presents environmental conditions rather aggressive for pedestrians and cyclists; moreover, almost all buildings on campus are detached from the road, significantly weakening its attractiveness.

The proposed realization of a continuous cycle/pedestrian path (col. 2, scenario A) that runs well beyond the distribution road and links together all activity centres and the most central systems of places on Campus is blessed by a great centrality potential for all indexes. Most important, the new path is continuously central, with no significant local drops, weakening the previously dominant centrality system of the distribution road. In a way, the new path effectively "drains" centrality flows over the system, while at the same time resulting mostly consistent with the structure of places' centrality (fig. 3).

The alternative proposal (col. 3, scenario B) does not exhibit a comparable success. The idea of building a ring that includes part of the distribution road lacks an acceptable level of centrality especially in the north-eastern side, with particular reference to $\mathrm{B}^{\mathrm{C}}$ and $\mathrm{I}^{\mathrm{C}}$. The realization of such a ring would probably lead to a more confused system and a certain waste of financial and territorial resources. 
Thus, the leading aim of the project has been defined to create a new dorsal cycle/pedestrian path that intersects and links all "local centers" running on an internal route. The new dorsal path should be connected to the external vehicular parking-roads in order to favor the car/bicycle exchange.

After a detailed investigation of functions, place centralities and the conditions of existent paths to be reconnected, two alternative scenarios were defined and analyzed again through a process of MCA. The two scenarios (fig. 4, thick black) especially differ in the eastern side of the Campus, where the first is spine-shaped and the second follows a more ring-like route.

The results of the MCA analysis over these two alternative networks are presented in fig. 5 , col. 2 and 3 . ently than in dual generalized analysis like those of Space Syntax, the continuity of a central route is not the outcome of a generalization model and its distinct rationale, but rather the outcome of the "natural flow" of centralities over each edge throughout the real architecture of connections and the metric disposition of spatial components in the system.

A rather worse performance is offered by the ringshaped scenario B. Centralities describe a fragmented interrupted route that do not assume a univocal leading role in the system. The image that emerges, under this scenario, is that of a system that shows at least three routes at the same level, none of them really continuous, each with a certain level of contradiction between the four different indexes of centrality.

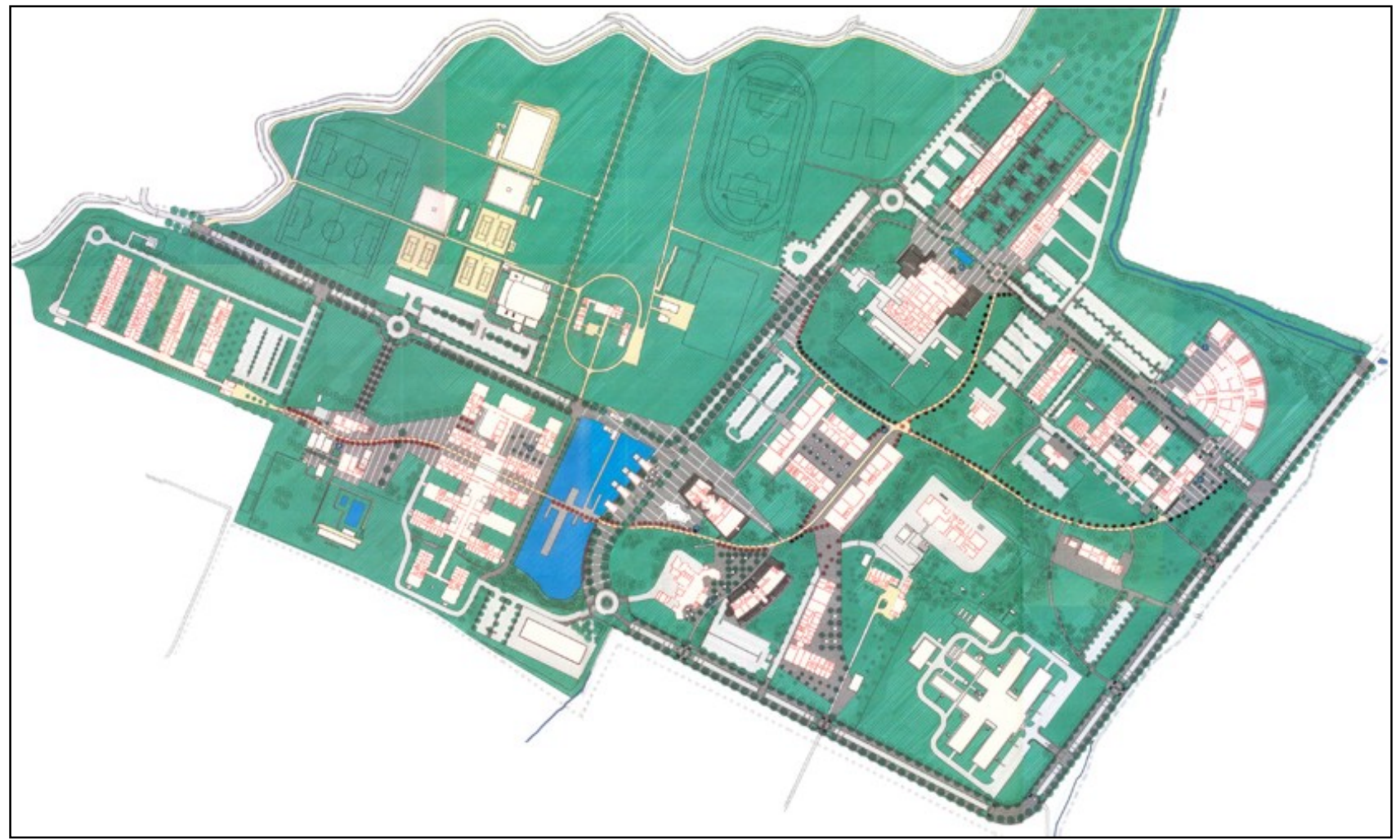

Fig. 6

The final layout of the urban design plan for the Campus. The cycle/pedestrian spine is clearly visible that connects all local cenralities; the core of the campus is landscaped with a new paved area and a small lake which is bridged by the spine through a wooden platform. All streets are traffic-clamed, though at a different degree taking into account the role of each of them in the general model of accessibility. Parking areas are largely substituted by on-street parking. Long-term parking has been placed along the external service street. The plan takes advantage of the implementation of MCA analysis.

MCA reveals that the proposed scenario A, the spineshaped route that crosses the eastern side of the Campus with two other branches departing from the Scientific Engineering Department towards the sport plants and the Pharmacy Department, scores very well with all centrality indexes. This route, that is obtained by the reconnection of existent but fragmented path segments through new paths, actually achieves a leading role in the whole system, leaving lower results to the currently dominant route of along the distribution road.

Most important, the new spine route emerges as a continuous central systems with no significant interruptions. This result is highly significant because in MCA, differ-
In-depth investigations of local conditions have been implemented by specific MCA analysis as well, but basically the successive steps have been oriented to a classic work of urban design on the basis of the realization of the spine-shaped new cycle-pedestrian route across the Campus. The new path has been detailed with a new lightning system, new paving and a continuous line of blackthorns that gives the path - now called "the blackthorns way" - a distinctive and unique identity, and the quality of open spaces in local centres has been particularly enhanced.

Traffic calming techniques have been implemented over all roads, with distinct characteristics between the internal distributor and the external parking road. New landscaped 
areas have been designed where large surfaces of existing parking lots have been cancelled. A system of cycle free rental has been provided at interchanges between the car and the pedestrian oriented environments. Architectural solutions have been proposed in some specific spots, i.e. where the "blackthorns way" passes through the main corridor of the Didactic Engineering Department: here, a new atrium has been created that realizes a long invoked new entrance to the building. A core of high quality open spaces has been individuated for the whole Campus adjacent to the recently built administrative centre, that involves landscaped as well as paved areas: a new lake has been designed that is crossed by the "blackthorns way" through a long wooden bridge, and a former rugby facility has been displaced in order to realize a new area for events and celebrations just in front of the lake. A landscaped park has been finally proposed along the sidebranches of the new "blackthorns way".

\section{Conclusions}

The Multiple Centrality Assessment (MCA) has proved greatly helpful in orienting a project of urban design across both the problem setting and problem solving phases. In problem setting, MCA provided a new understanding of the reciprocal relationships between two different spatial systems, that of places and that of cycle/pedestrian paths; both systems belong to the more general system of the open spaces, so the emerging inconsistencies have been of full interest for the urban designer.

In the Campus "Area of the Sciences" in Parma, the main problem is due to the different structures of the two systems that makes the marginal paths serve the central places and the central paths serve the marginal places. A deep reform of the network of paths has been addressed in the problem solving phase: two alternative solutions have been tested again through a process of MCA. The most convincing of the two scenarios, a spine-shaped new cycle/pedestrian route that connects the most central subareas and the main centers of activities on Campus, well detached from the distributor road, has been detailed in the project of urban design that touched, on that basis, a wide range of spatial and functional issues.

In the context of this process of urban design, MCA provided a valuable contribution because of its distinctive characteristic of being based on a set of four different centrality index, a primal graph representation of complex geographic networks and a metric computation of distances. The result is a multifaceted, argumentative understanding that does not offer any single, apodictic, universal key to all possible problems of open spaces, but rather an in-depth description of the different properties of the newtork in question, properties that belong to different ways of being central. All these properties, all these different ways of being central, do work at the same time in a complex geographic system, with a deep impact on how people and human activities distribute spatially over the system itself. But the "behavior" of those properties and the level of reciprocal consistency depends on local conditions, topological as well as geographical.
Finally, we do not think that the network analysis of a complex spatial system, even so multifaceted and sophisticated as MCA, can tell the whole story. Complexity cannot be reduced to one dimension, even to such an inclusive one like the geo-topological dimension of the spatial newtork. MCA just offers a point of view. An extremely enlightening one, to be true, but just one of the many that contribute to the astonishing, ever changing, surprising world of the social use of public open spaces.

\section{References}

[1] Watts D J, Strogatz S H, 1998, "Collective dynamics of small-world networks", Nature 393 440-442.

[2] Albert R, Barabási A L, 2002, "Statistical Mechanics of Complex networks", Review of Modern Physics 74 47-97.

[3] Wasserman S, Faust K, 1994, Social Networks analysis (Cambridge University Press, Cambridge, UK).

[4] Bavelas A, 1948, "A mathematical model for group structures", $\mathrm{Hu}$ man Organization 7 16-30.

[5] Bavelas A, 1950, "Communication patterns in task oriented groups", Journal of the Acoustical Society of America 22 271-282.

[6] Wilson GA, 2000, Complex Spatial Systems: The Modelling Foundations of Urban and Regional Analysis (Prentice Hall, Upper Saddle River, NJ).

[7] Hillier B, Hanson J, 1984, The social logic of space (Cambridge University Press, Cambridge, UK).

[8] Hillier B, 1996, Space is the machine: a configurational theory of architecture (Cambridge University Press, Cambridge, UK).

[9] Salheen M, Forsyth L, 2001, "Addressing distance in the Space Syntax syntactical model”, Urban Design International 6 93-106.

[10] Ratti C, 2004, "Space Syntax: some inconsistencies", Environment and Planning $B \mathbf{3 1}$ 513-516.

[11] Porta S, Crucitti P, Latora V, 2004, "The network analysis of urban streets: a dual approach", pre-print cond-mat/ 0411241.

[12] Crucitti P, Latora V, Porta S, 2005, "Centrality measures in urban networks", in preparation.

[13] Porta S, Crucitti P, Latora V, 2005, "The network analysis of urban streets: a primal approach", in preparation.

[14] Porta S, Crucitti P, Latora V, 2004, "The network analysis of urban streets: a dual approach", preprint cond-mat/ 0411241.

[15] Hillier B, 1996, Space is the machine: a configurational theory of architecture (Cambridge University Press, Cambridge, UK).

[16] Dalton N, Peponis J, Dalton R, 2003, "To tame a tiger one has to know its nature: extending weighted angular integration analysis to the description of GIS road-centerline data for large scale urban analysis", Proceedings 4th International Space Syntax Symposium, London, UK.

[17] Jiang B, Claramunt C, 2002, "Integration of Space Syntax into GIS: new perspectives for urban morphology", Transactions in GIS 6 $151-162$.

[18] Jiang B, Claramunt C, 2004, "A structural approach to the model generalization of an urban street network", GeoInformatica 8 157171 . 